\title{
Permanent pacing in a patient with a left ventricular assist system
}

\author{
M H Cave, J Wallwork, P M Schofield
}

\begin{abstract}
A patient with end stage heart failure underwent permanent implantation of a Novacor left ventricular assist system. Progressive right ventricular dysfunction developed during the first two postoperative weeks. Temporary and subsequently permanent atrioventricular pacing to correct first degree heart block improved the performance of the device, with sustained resolution of his right ventricular failure.
\end{abstract}

(Heart 1996;75:525-527)

Keywords: assist system; first degree heart block; right ventricular failure; atrioventricular pacing

The Novacor left ventricular assist system (LVAS) is an electrically driven implantable blood pump designed to work in parallel with the left ventricle, taking blood from the apex of the left ventricle and delivering it to the ascending aorta. ${ }^{1}$ The output of the device depends primarily on filling, which in turn is dependent upon delivery from the right side of the circulation, together with active filling from the native left ventricle. With a stroke volume of $70 \mathrm{ml}$, the device is capable of total left ventricular unloading. Peak LVAS fill rate and output are monitored on a beat to beat
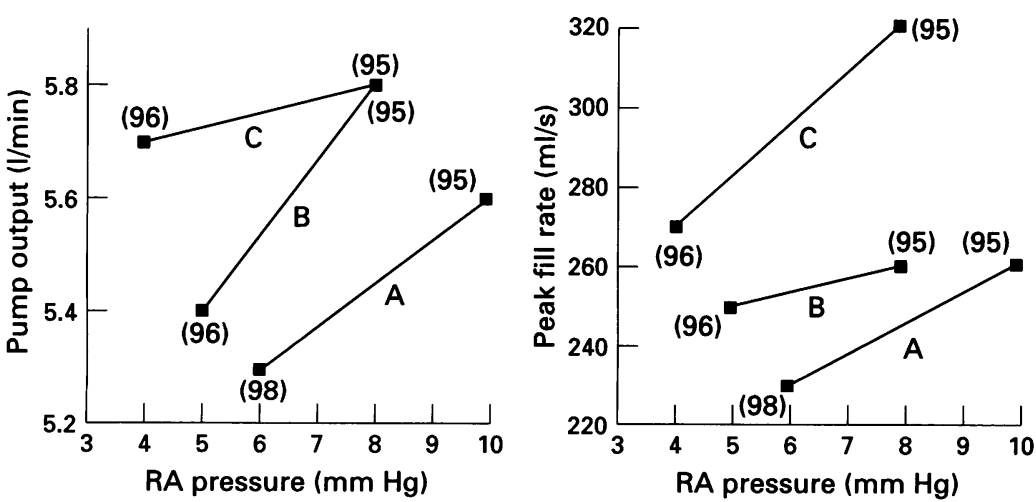

Figure 1 Effect on pump output and peak fill rate of adjusting the $A V$ interval. (A) sinus rhythm with PR interval of $220 \mathrm{~ms}$, (B) atrial tracking with an AV interval of 200 $m s$, (C) atrial tracking with an AV interval of $100 \mathrm{~ms}$. RA, right atrial. Figures in parentheses indicate heart rate. Note that the pump was running in fill-to-empty mode during these measurements, which means that the pump rate was not necessarily equal to the heart rate. basis, and these variables can be used as indirect indicators of right ventricular function.

\section{Case report}

A man of 62 with advanced ischaemic left ventricular failure underwent elective permanent implantation of a Novacor LVAS as part of a pilot study to compare the LVAS with medical treatment in patients with end stage heart failure. At preoperative assessment, echocardiography showed dilatation of the left and right ventricles with moderate functional mitral and tricuspid regurgitation. A resting 12 lead electrocardiogram showed sinus rhythm with a PR interval of $220 \mathrm{~ms}$, axis $-120^{\circ}$, right bundle branch block, and $Q$ waves in V1-4.

Implantation of the LVAS was uncomplicated. Perioperative transoesophageal echocardiography showed an increase in the severity of pre-existing tricuspid regurgitation with right atrial enlargement after the start of left ventricular assistance. During the second postoperative week the patient developed considerable peripheral oedema and an increase in jugular venous pressure with persistently reduced filling of the LVAS, indicating right ventricular failure. This was successfully treated with diuretics, inotropes, and atrioventricular (AV) sequential pacing.

It was noted that shortening the AV interval, without changing the heart rate, had a beneficial effect on LVAS function. Thus during sinus rhythm at 102 beats/min, with a PR interval of $220 \mathrm{~ms}$, the peak fill rate was 190 $\mathrm{ml} / \mathrm{min}$ and the pump output was $5.31 / \mathrm{min}$. Atrial tracking and ventricular pacing at the same sinus rate with an AV interval of $100 \mathrm{~ms}$ increased these variables to $230 \mathrm{ml} / \mathrm{min}$ and $6.2 \mathrm{l} / \mathrm{min}$ respectively. A Medtronics Thera-D dual chamber pacemaker was therefore inserted three weeks after LVAS implantation. Haemodynamic variables during various pacing modes were recorded in the pacing laboratory, with the patient supine and at $30^{\circ}$ feet-down tilt to alter venous return. Figure 1 shows how shortening the AV interval enhanced LVAS (and hence right ventricular) performance. This electrophysiological manoeuvre also decreased the systolic wave on the right atrial pressure trace (fig 2). On the basis of these data, the pacemaker was programmed to DDD mode with a base rate of 90 beats $/ \mathrm{min}$ 


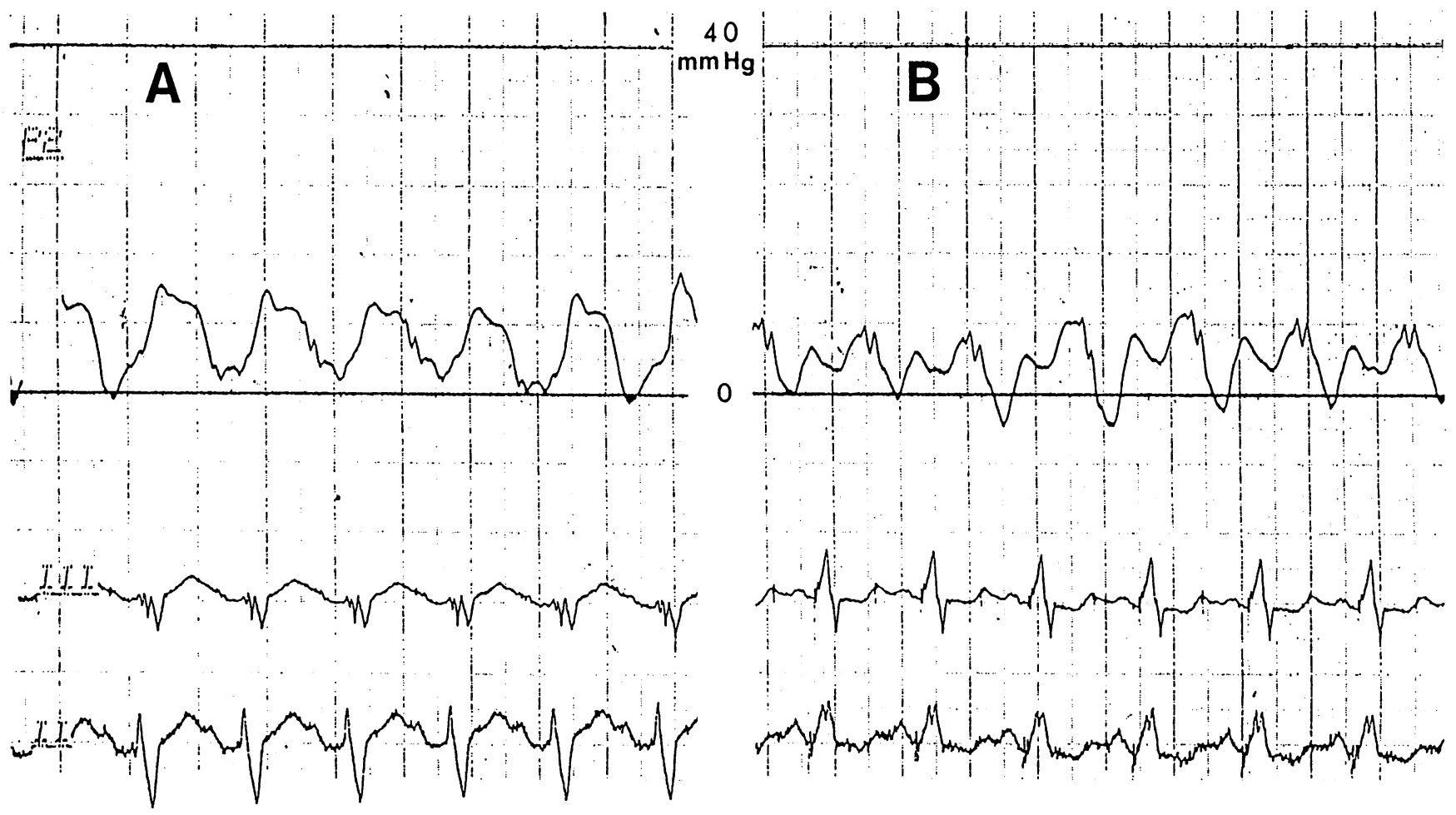

Figure 2 Right atrial pressure (upper trace) with and without $A V$ sequential pacing. (A) sinus rhythm, no pacing; (B) sinus rhythm with atrial tracking, AV interval $100 \mathrm{~ms}$. Calibration of the pressure trace was unchanged between recordings.

and an AV interval (paced and sensed) of 120 ms.

The patient progressed well with no signs of heart failure on no diuretic therapy. At the time of discharge from hospital, six weeks after LVAS implantation, the beneficial haemodynamic effect of reducing the AV interval was again demonstrated. On this occasion, transthoracic echocardiographic colour Doppler mapping suggested a decrease in the magnitude of the tricuspid regurgitant jet during the transition from sinus rhythm to atrial tracking.

\section{Discussion}

First degree heart block is a common, benign conduction defect that usually has no overt haemodynamic consequences, even in patients with severe heart disease. In this patient, however, the use of DDD pacing to correct first degree block had a beneficial effect on haemodynamic indices of right ventricular performance, as reflected by the filling characteristics and output of the LVAS, and contributed to the resolution of right ventricular failure after LVAS implantation.

In most patients, pulmonary vascular resistance decreases and systemic venous return increases after LVAS implantation, with an improvement in right ventricular function. ${ }^{2}$ However, acute right ventricular failure occurs in about $20 \%$ of patients, ${ }^{3}$ although the insidious onset observed in our patient was unusual. The mechanism of right ventricular failure after mechanical left ventricular support is probably multifactorial. One theory is that left ventricular unloading causes a leftward shift of the interventricular septum. ${ }^{4}$ This interferes with right ventricular contraction and, in association with increased venous return, encour- ages functional tricuspid regurgitation, ${ }^{5}$ which appeared to worsen after LVAS implantation in this case. Delayed ventricular systole caused by first degree heart block could exacerbate this abnormality, and this may be the mechanism by which reducing the $A V$ interval improved right ventricular function in this patient. The right atrial pressure recordings and echocardiographic findings support this theory. Another possibility is that right ventricular contraction was more efficient when initiated by pacing rather than conduction of sinus beats with right bundle branch block. This may explain the haemodynamic improvement induced by atrial tracking and ventricular pacing with the AV interval reduced only slightly to $200 \mathrm{~ms}$ (line B in figure 1).

To our knowledge, permanent pacing has not previously been used in a patient with an LVAS, which, until now, has only been used after severe post-cardiotomy shock or as a bridge to cardiac transplantation. If the LVAS proves to be a successful long term treatment in severe heart failure, AV sequential pacing may have a place in the management in these patients, many of whom will have apparently unimportant conducting system disease. We can only speculate on the possible implications in other patients in whom right ventricular function is impaired but who do not have the anatomical abnormalities unique to LVAS patients.

We thank DW Wheeldon and JA Smith for their help in the preparation of this report.

1 McCarthy PM, Portner PM, Tobler HG, Starnes VA, Ramasamy N, Oyer PE. Clinical experience with the Ramasamy N, Oyer PE. Clinical experience with the Surg 1991;102:578-87. 
2 Bennick GBWE, Noda H, Duncan JM, Frazier OH. Clinical evaluation of right ventricular function in patients with left ventricular assist device (LVAD). Int $\mathscr{f}$ Artif Organs 1992;15:109-13.

3 Frazier OH, Rose EA, MacManus Q, Burton NA, Lefrak EA, Poirier VL, et al. Multicenter clinical evaluation of EA, Poirier VL, et al. Multicenter clinical evaluation of The Heartmate 1000 iP left vent
4 Chow E, Farrar DJ. Right heart function during prosthetic left ventricular assistance in a porcine model of congestive heart failure. I Thorac Cardiovasc Surg 1992; 104:569-78.

5 Holman WL, Bourge RC, Fan P, Kirklin JK, Pacifico AD, Nanda NC. Influence of left ventricular assist on valvular regurgitation. Circulation 1993;88(part 2):309-18.

\section{ABSTRACTS IN CARDIOLOGY}

\section{Investigation of unexplained left ventricular hypertrophy}

The genes responsible for unexplained ventricular hypertrophy are slowly being recognised. Abnormal fibrillary proteins including desmin, heavy chain myosin, tropomyosin, and troponin are responsible for the phenotype we call hypertrophic cardiomyopathy. A very similar cardiac morphology can be produced by storage disorders with glycogen or galactose but systemic abnormalities are present to point to the diagnosis. The study reported below, however, shows that among 270 patients with echocardiographic left ventricular hypertrophy without any systemic dis- order seven had plasma and lymphocyte activity of $\alpha$-galactosidase enzyme far below the normal range. Cardiac biopsy and study of the gene confirmed heterozygous Fabry's disease. Subclinical manifestation of Fabry's disease therefore has to be considered in the differential diagnosis of unexplained left ventricular hypertrophy but this involves either cardiac biopsy with electron microscopy or assay of plasma concentrations of an enzyme that is not likely to figure in the tests offered by most laboratories.

M J DAVIES

\section{An atypical variant of Fabry's disease in men with left ventricular hypertrophy}

Shoichiro Nakao, Toshihiro Takenaka, Masato Maeda, Chihaya Kodama, Akihiro Tanaka, Minoru Tahara, Aichi Yoshida, Masaru Kuriyama, Hidemasa Hayashibe, Hitoshi Sakuraba, Hiromitsu Tanaka

Bachground-Fabry's disease is an X-linked recessive disorder that results from a deficiency of $\alpha$-galactosidase. Left ventricular hypertrophy is one of the common manifestations in men with classic hemizygous disease. Recently, several cases of an atypical variant of hemizygous Fabry's disease, with manifestations limited to the heart, have been reported. Therefore, we assessed the incidence of hemizygosity for Fabry's disease among male patients with left ventricular hypertrophy.

Methods-We measured plasma $\boldsymbol{\alpha}$-galactosidase activity in 230 consecutive male patients with left ventricular hypertrophy. Clinical manifestations were assessed, endomyocardial biopsies were performed, and the patients were screened for mutations in the $\alpha$-galactosidase gene.

Results-Seven of the 230 patients with left ventricular hypertrophy ( 3 percent) had low plasma $\alpha$ galactosidase activity ( 4 to 14 percent of the mean value in normal controls). These seven unrelated patients, ranging in age from 55 to 72 years, did not have angiokeratoma, acroparesthesias, hypohidrosis, or corneal opacities, which are typical manifestations of Fabry's disease. Endomyocardial biopsy was performed in five patients and revealed marked sarcoplasmic vacuolization in all five. Samples from four patients were examined by electron microscopy and revealed typical lysosomal inclusions with a concentric lamellar configuration in all four. Two patients had novel missense mutations in exon 1 and exon 6 . The remaining five had no mutations in the coding region of the $\alpha$-galactosidase gene, but the amounts of the o-galactosidase messenger RNA were markedly lower than normal.

Conchusions - Seven unrelated patients with atypical variants of hemizygous Fabry's disease were found among 230 men with left ventricular hypertrophy. Fabry's disease should be considered as a cause of unexplained left ventricular hypertrophy. (N Engl I Med 1995;333:288-93.) 